\title{
Somatotopy of monkey premotor cortex examined with microstimulation
}

\author{
Moshe Godschalk*a, Andrew R. Mitz ${ }^{\mathrm{b}}$, Berber van Duin ${ }^{\mathrm{a}}$, Hans van der Burg ${ }^{\mathrm{a}}$ \\ ${ }^{a}$ Department of Anatomy, Faculty of Medicine and Health Sciences, Erasmus University Rotterdam, P.O. Box 1738, 3000 DR Rotterdam. \\ The Netherlands \\ ${ }^{b}$ Laboratory of Systems Neuroscience, National Institute of Mental Health, NIH Animal Center, P.O. Box 608, \\ Poolesville, MD 20837-0608, USA
}

Received 30 September 1994; revision received 17 July 1995; accepted 19 July 1995

\begin{abstract}
We reinvestigated the organization of the premotor cortex (PM) using intracortical microstimulation. Movements of forelimb, hindlimb, and orofacial structures were evoked from broad regions of PM that appeared to be contiguous with other motor areas. There were two principal findings: (1) the somatotopy of PM lies roughly parallel to that of the primary motor cortex (MI). Forelimb movements were evoked from sites deep in the caudal bank of the arcuate sulcus and throughout the adjacent cortex bounded by a face representation (laterally) and a hindlimb representation (medially and caudally); (2) unlike the MI, the PM forelimb representation overlaps significantly with its own face representation. PM hindlimb movement sites overlap only slightly with PM forelimb sites, in a manner similar to the MI. There was no obvious boundary between PM, MI, or supplementary motor area hindlimb representations. The present findings are discussed in relation to recently identified subdivisions of the PM.
\end{abstract}

Keywords: Monkey; Premotor cortex; Somatotopy; Microstimulation

\section{Introduction}

Efforts to segregate the primary motor cortex (MI) into different functional subdivisions have met with only limited success (Strick and Preston, 1982a,b; Humphrey and Reed, 1983; Aizawa et al., 1990). In contrast, the non-primary motor areas have experienced a recent spate of subdivisions. The frontal eye field (FEF) has separate regions for saccadic and smooth pursuit eye movements (Gottlieb et al., 1993). The supplementary motor area (SMA) has at least two different forelimb movement areas: a caudal one (SMA) specialized for internally-guided actions (e.g., using a remembered sequence) and a slightly more rostral one (pre-SMA) specialized for externally-guided actions (e.g., using a visual cue) (Shima et al., 1991; Shima and Tanji, 1994), as well as its own supplementary eye field (SEF, Schlag and Schlag-Rey, 1987). The premotor cortex (PM) has a separate dorsal area (PMd), where pre-movement 'set' re-

* Corresponding author. Tel.: +31 10408 7298; Fax: +31 10436 5780; E-mail: godschalk@anat.fgg.eur.nl. lated activity is more common, and a ventral (PMv) area, where movement-related activity dominates (Mushiake et al., 1991; Kurata, 1993). PMv appears to have at least two different specialized areas of its own. One part of PMv, just caudal to the genu of the arcuate sulcus, has units that are related to both the hand and face (Gentilucci et al., 1988), some of which operate in a face-centered coordinate system (Fogassi et al., 1992) and others of which operate in a hand-centered coordinate system (Graziano and Gross, 1994). The other part of the PMv, more lateral, has units that appear to encode grip force similar to units in the MI (HeppReymond et al., 1994). Unlike the SMA, it remains unclear where the various subdivisions of the PM lie in the overall somatotopic architecture of the PM, or if the PM has zero, one, or more complete somatotopic map(s). PM connections with the MI indicate that its somatotopy is roughly like that of the MI (Matsumura and Kubota, 1979; Muakkassa and Strick, 1979; Godschalk et al., 1984; see also Morecraft and Van Hoesen, 1992). Single-unit recordings have generally supported this view (Kurata et al., 1985; Gentilucci et 
al., 1988; Rizzolatti et al., 1988; Kurata, 1989). However, Muakkassa and Strick (1979) suggest that the PM may have multiple body representations because relatively discrete injections of horseradish peroxidase into the the MI hand representation labels more than one circumscribed region of the PM. Their findings were similar for the hindlimb area projections. Other anatomical results are more reflective of a single representation in the PM (Godschalk et al., 1984; Dum and Strick, 1991; Morecraft and Van Hoesen, 1992). If there is only a single representation, it may be very diffuse. Using a physiologically-controlled injection of diamidino yellow into the forelimb area of the SMA, Luppino et al. (1990) labeled a relatively wide expanse of the $P M$, much more extensive than the forelimb area(s) of the PM as described by Muakkassa and Strick (1979). A diffuse representation is also suggested by the apparent lack of forelimb versus hindlimb segregation observed with cortical field potential recordings (Hashimoto et al., 1981).

The roles for the separate subdivisions of the PM cannot be fully assessed until there is a greater understanding of the PM somatotopy. One impediment to viewing the PM somatotopy has been the relative paucity of movements evoked with standard microstimulation methods in this area (Weinrich and Wise, 1982; Wise and Mauritz, 1985; Gentilucci et al., 1988). We decided to apply a modified microstimulation method, one that has been effective in the SMA and the adjacent cortex (Mitz and Wise, 1987; Mitz and Godschalk, 1989), to reexamine PM somatotopic organization. Some of these data have been reported in abstract form (Godschalk et al., 1990).

\section{Methods}

\subsection{Subjects and materials}

Two Macaca nemestrina, a $6.8 \mathrm{~kg}$ female (M57) and a $7.6 \mathrm{~kg}$ male (M58) were used in this study. The monkeys were adapted to the apparatus before implantation. Each animal was anesthetized twice, once for skull measurements and the second time for implantation of a custom stainless steel head restraint and chronic chamber, as detailed elsewhere (Lemon, 1984). Each chamber was centered over the left arcuate sulcus.

Epoxylite-coated tungsten wire electrodes were used for both stimulation and recording. The exposed metal tip dimensions of fresh electrodes could not be clearly identified with light microscopy; in saline they had impedances around $100 \mathrm{k \Omega}$ at $1 \mathrm{kHz}$. During a penetration the impedance dropped gradually to about $30 \mathrm{k} \Omega$. Afterwards, the exposed tip was visible with a microscope, and measured from 20 to $100 \mu \mathrm{m}$ along the long axis.

A differential amplifier recorded unit potentials between the electrode and the implant. This signal was amplified and displayed on an oscilloscope. The same signal was filtered, passed through adjustable limiters, and discriminated with a voltage level discriminator. A remote switch grounded the differential amplifier during stimulation and connected the stimulating pod to the electrode. The pod included a biphasic constant-current stimulator (Mitz et al., 1984), a wide bandwidth current monitor, and a high compliance stimulus isolation unit. Trains of 31 biphasic pulses were delivered at 330 pulses/s. Each phase was $0.2 \mathrm{~ms}$ in duration (cathodal first) and the two phases were symmetrical in amplitude. The current was usually limited to $60 \mu \mathrm{A}$ for either phase. A digital storage oscilloscope (Phillips PM3302) was used to monitor the shapes and amplitudes of the current pulses. Electrode impedance was calculated from current flow created by a $1 \mathrm{~V}$ p-p sine wave $(1 \mathrm{kHz})$ imposed across the electrode in vivo.

\subsection{Data collection}

Up to five penetrations were made each day during 1-4 h sessions, but no penetrations were within $1 \mathrm{~mm}$ of each other on a given day. A strict protocol was employed for every penetration. The electrode was lowered manually to establish contact with the dura. Next, a hydraulic microdrive was used for further movement. Once the electrode had entered the brain, as judged by the presence of unitary potentials, the electrode was raised until the activity diminished. This relatively superficial, quiet site was taken as the start of the electrode track. After $30 \mathrm{~s}$ the electrode was lowered in 200-250 $\mu \mathrm{m}$ steps, isolating single units whenever possible. Notes were made on the size and degree of unit activity at the end of the step, and then the site was stimulated, beginning with $60 \mu \mathrm{A}$ of current for either phase. During stimulation the monkey was visually scanned by at least two experienced observers. The mechanical arrangement of the monkey 'chair' permitted easy observation of the entire animal, and the entire animal was scanned during stimulation at each site. The thresholds for each movement evoked at $60 \mu \mathrm{A}$ were assessed by a descending series of currents; threshold was defined as the lowest current with which a movement was evoked by at least $50 \%$ of the delivered stimuli. On rare occasions muscles were palpated to help identify a movement; no electronic recording methods were used. Movements were defined as a rotation about a joint, other localized movement of a body part (e.g., eye rotation, scalp movement), or the contraction of muscles that would cause a movement if that body part were free to move (e.g., neck muscle contraction with the head restrained). We did not look for smoothpursuit eye movements; all recorded eye movements were saccadic. Stabilizing contractions and movements of unexposed body parts (e.g., larynx) were not identified and were not recorded as movements.

\subsection{Data analysis and histology}

The animal was overdosed with sodium pentobarbital 
$(200 \mathrm{mg} / \mathrm{kg}$ ) and perfused through the heart with saline followed by a buffered $4 \%$ formaldehyde solution. The brain was removed and sectioned along the coronal plane at $\mathbf{4 0} \mu \mathrm{m}$ with a freezing microtome. Every second section was mounted, stained for Nissl substance with cresyl violet, and inspected microscopically.

Every second stained section was projected at $20 \times$ magnification to make detailed drawings of the anatomy. After identifying or estimating the electrode trajectory for each penetration (see below), representative sections were selected from this initial set of drawings to plot evoked movement data. The representative sections were spaced as necessary to reproduce sulcal landmarks and stimulation data (between 320 and $800 \mu \mathrm{m}$ apart). The representative sections were then redrawn (Fig. 1) according to a reconstruction technique that follows the principles described by Van Essen and Maunsell (1980) based on a flattened three-dimensional wire model. The projection of all data to cortical layer $\mathrm{V}$ is a convenient formulation that also reflects the method's inability to reconstruct cortical depth profiles along non-radial tracks. No assumption is made regarding somatotopic organization in the radial dimension (e.g., columnar organization) by this diagrammatic approach. In a few cases data were collected from different layers along the same radial 'column' of cortex; the lowest observed threshold for each evoked movement was selected for analysis. These cases were infrequent, and multiple electrode tracks through the same region of cortex at different layers were also infrequent. Errors in penetration angle measurements (see below) and electrode depth measurements further impeded reconstructions of cortical 'columns' using data from different electrode tracks. Because of all these factors, no attempt was made to analyze thresholds or movements as a function of the cortical layer.

Patterns of somatotopic organization (Fig. 3) are based on the above reconstructions. The patterns are constructed by identifying a border for each characteristic of interest: the border connects cortical sites where the relevant response was evoked, without including sites where the relevant response was not evoked. This rule allows the inclusion of a few, isolated, unexplored sites within an organizational pattern. To this degree, the pattern does not exactly represent the data. Larger unexplored or unresponsive regions are represented as 'holes' in an organizational pattern.

Special consideration was given to preventing errors in penetration trajectories and penetration depths that could cause large errors in the final location of stimulation sites on the two-dimensional maps. The positions of stimulation sites along a penetration track were assigned based upon microdrive readings, and the evaluation of single unit potential shapes and amplitudes recorded at each site before stimulation. Unit recordings provided estimates of the locations of gray and white matter tran- sitions. Marking lesions were used to estimate and compensate for shrinkage due to fixation. The vast majority of penetration tracks ( $70 \%$ in M57, $82 \%$ in M58) were identified by gliosis in the histological material (recovered tracks). Many of the remaining tracks ( $10 \%$ of all tracks in M57, 8\% of all tracks in M58) were recovered based on gliosis that occurred along only part of each track. The trajectories of the remaining electrode tracks (20\% in M57, $10 \%$ in M58) were estimated based on chamber coordinates, marking lesions, and angle measurements of nearby tracks. Angles were measured relative to the sagittal plane. To assess the accuracy of the estimated trajectories, we evaluated the distribution of angles of the recovered tracks from M58 $(n=145)$. This was fit to a Gaussian distribution (mean $=17.92$, S.D. $=4.52$ ). The absolute error introduced if we had used just the distribution mean as a trajectory angle estimator was 3.44 degrees (S.D. $=2.91$ ). However, because the angles varied almost linearly with rostrocaudal position as well as with mediolateral position, we used only nearby penetrations to estimate trajectory angles. To estimate the error from this latter approach, we used a linear multiple regression model (estimated penetration angle $=12.15+(0.23 \times$ mediolateral coordinate $)+(0.14 \times$ rostrocaudal coordinate $))$. These errors were slightly less $(3.23$ degrees, S.D. $=2.71$ ) than errors associated with using mean track angle as an estimator. To insure that the estimated trajectories did not lead to major errors in the final two-dimensional maps, we looked for any case where an estimated trajectory within the $99 \%$ confidence limit (3.82 degrees of angle, or $0.67 \mathrm{~mm}$ of error orthogonal to the predicted path per $10 \mathrm{~mm}$ of depth) could cause more than $2.0 \mathrm{~mm}$ error in the final assignment of a response site on the flattened cortical maps. All such ambiguous data points were discarded.

\section{Results}

\subsection{Distribution of movements}

For the purpose of description we take the Ml to be the region of the cortex between the fundus of the central sulcus and the rostral limit of the prevalent largest layer $\mathrm{V}$ pyramidal cells (Fig. 1). This criterion matches the physiological boundary between the MI and the PM, at least for the forelimb representation (Sessle and Wiesendanger, 1982; Weinrich and Wise, 1982), and it matches the physiological boundary between the MI and the SMA along the dorsomedial wall of the hemisphere (Wise and Tanji, 1981; Mitz and Wise, 1987). This definition is not useful laterally in the MI, where large layer $\mathrm{V}$ pyramidal cells are rare. Further, careful exploration of this lateral region has failed to identify a clear boundary between the MI and the PM (McGuinness et al., 1980). The SMA is defined as previously (Mitz and Wise, 1987): the excitable portion of the dor- 

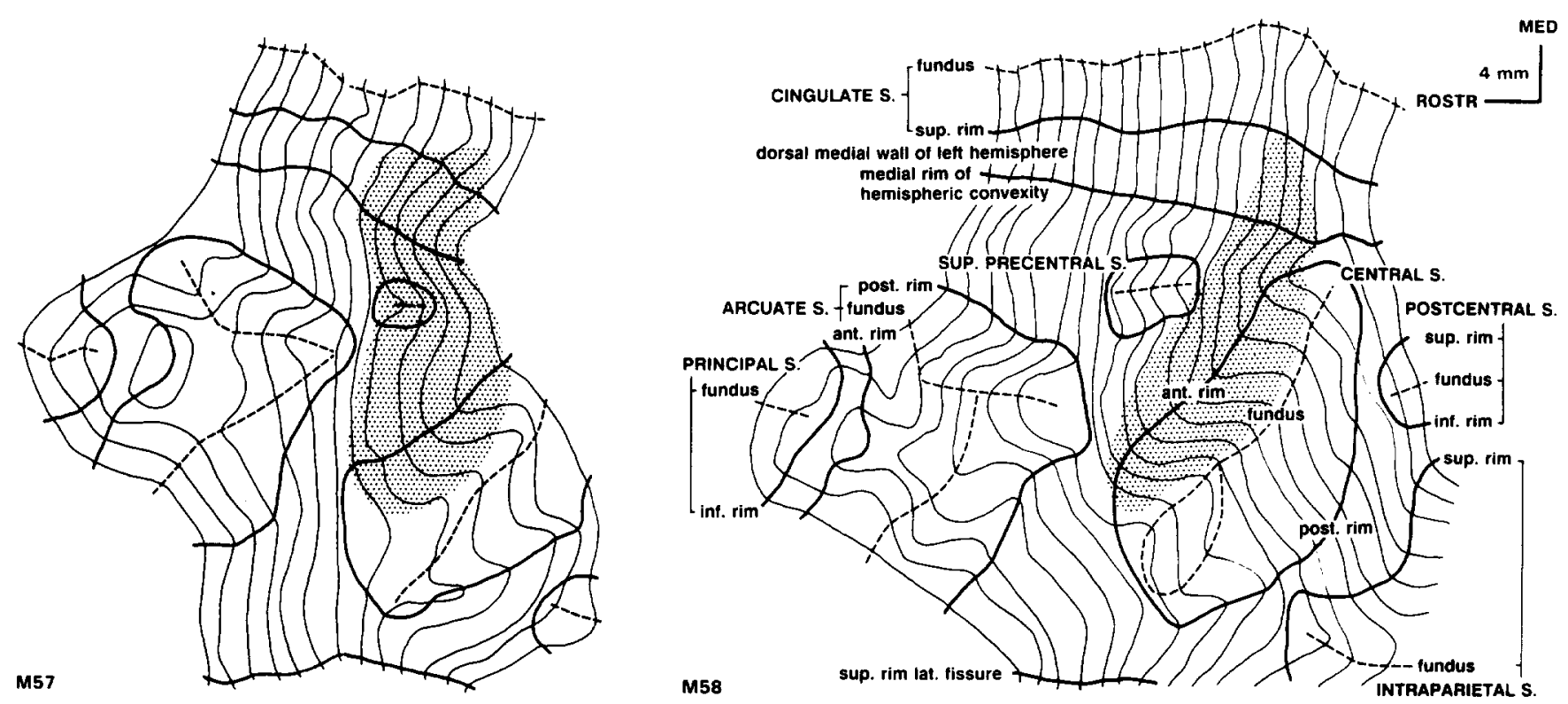

Fig. 1. Unfolded maps of the explored region in the frontal lobe of M57 (left) and M58 (right). Rostral is to the left, medial is up. Thick lines represent the lips of the sulci and dashed lines are positioned at the fundi. Thinner lines are isometric lines that follow the pyramidal cell layer (layer V) of representative sections. The stippled area marks where the largest layer V pyramidal (Betz) cells were prevalent. Key: s., sulcus; sup., superior; inf., inferior; ant., anterior; post., posterior; lat., lateral.

sal bank of the cingulate sulcus, the adjacent medial wall, and the nearby convexity of the hemisphere, that extends rostrally into a region where saccadic eye movements can be evoked and bounded caudally by large layer $\mathrm{V}$ pyramidal cells on the dorsomedial wall of the hemisphere. The caudal boundary is at or near the tail representation. The PM is assumed to be the cortex rostral to the MI and lateral to the forelimb representation of the SMA, bordered rostrally by the fundus of the arcuate sulcus. In the region between the dorsal limb of the arcuate sulcus and the SMA, the caudal-most evoked eye movement sites were used to mark the rostral boundary of the PM. This boundary is in accordance with the observation by Luppino et al. (1991), that these eye movement sites are located in area F7 as described by Matelli et al. (1991). F7 may also be the dysgranular cortex described by Di Pellegrino and Wise (1991).

The PM was the focus of the stimulation. Some additional mapping of other motor areas (e.g., MI, SMA) was included to accurately locate the PM relative to these other areas, and as a general guide for comparisons to previous, more detailed work in these other areas (see Discussion). We were able to evoke movements with less than $50 \mu \mathrm{A}$ from nearly all of the 107 penetrations into the cortex of M57 and 177 penetrations in M58. The area of exploration in M57 included much of the cortex bounded by the fundus of the central sulcus and the caudal tip of the principal sulcus, the midline, and the ventrolateral limit of the arcuate sulcus
(Fig. 2, left). The medial exploration covered parts of the dorsomedial wall of the hemisphere and the dorsal bank of the cingulate sulcus. Relatively fewer penetrations were made into the precentral convexity near the central sulcus. Exploration of M58 was similar, but with more sites in the central sulcus, extending into the posterior bank, and a less extensive survey of the cingulate sulcus (Fig. 2, right).

In both animals the explored region covered a substantial portion of the agranular frontal cortex including much of the MI, PM, and SMA. The general pattern of limb, trunk, and orofacial movement sites typical of the MI (Woolsey et al., 1952; Kwan et al., 1978; others), and of the SMA (Woolsey et al., 1979; Mitz and Wise, 1987; Luppino et al., 1991), are visible in the present study (Figs. 2, 3). Because these areas were not the thrust of this study, they were not as thoroughly explored. Saccadic eye movements were evoked from the frontal eye fields (FEF) in M58 (Fig. 2, right) and from sites just medial to the FEF, which may be in the supplementary eye field (SEF, Schlag and Schlag-Rey, 1987; Huerta and Kaas, 1990) or the adjacent cortex (Mitz and Godschalk, 1989). In each monkey, one single site was found in the caudal bank of the arcuate sulcus where downward directed saccadic eye movements coincided with closure of the eyelids. Movements were evoked from nearly all regions of the premotor cortex, although there is an area just rostral to the superior precentral sulcus and just medial to the arcuate sulcus 

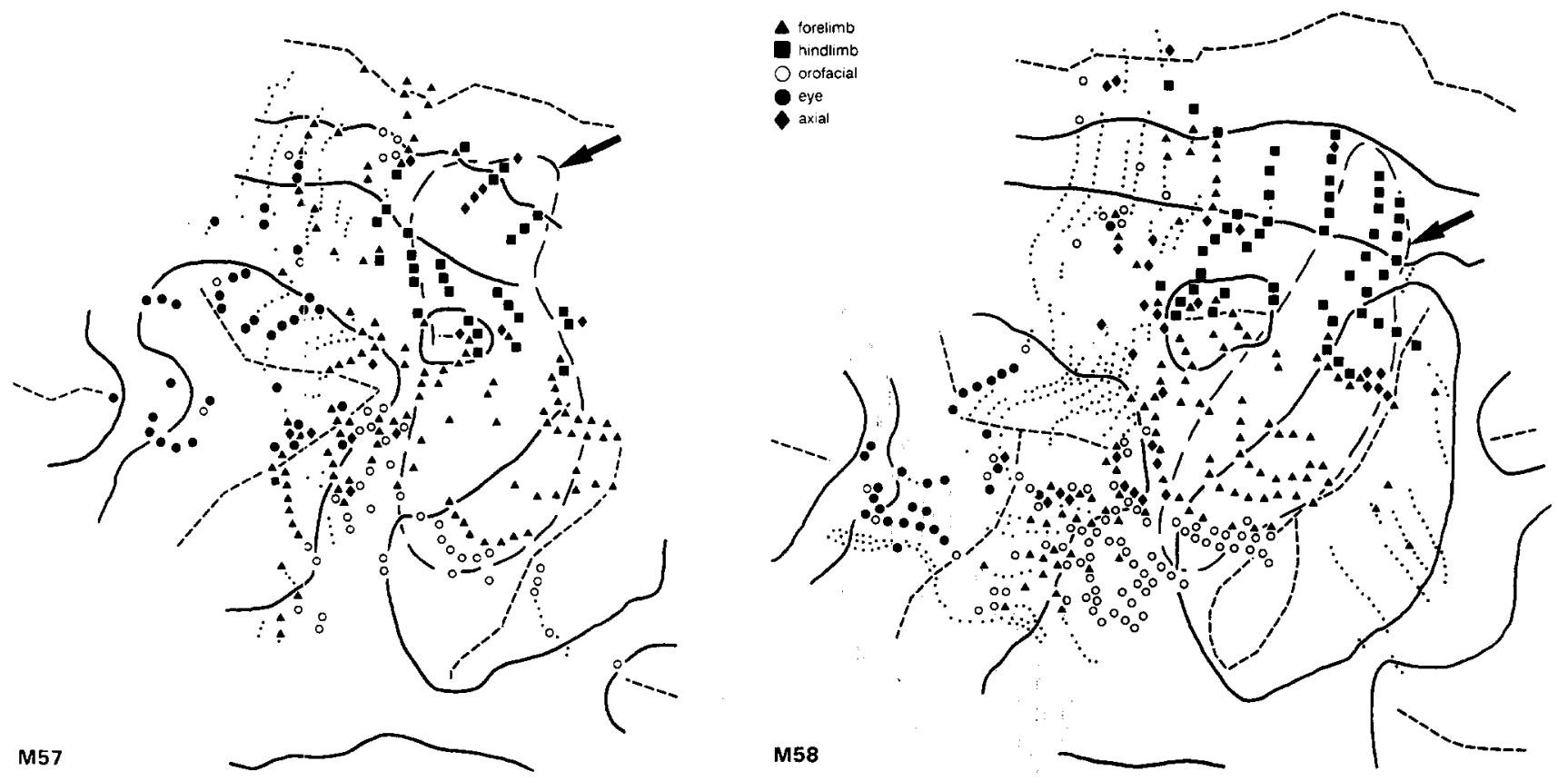

Fig. 2. Area explored by microstimulation at $60 \mu \mathrm{A}$ and distribution of evoked movements for stimulus currents at or below $50 \mu \mathrm{A}$. Orientation as in Fig. 1. Arrow in each drawing points to an outline of the region where Betz cells were identified (see Fig. 1). Small dots indicate stimulation sites where no movements were evoked. In a few cases adjacent symbols mark a single site where diverse movements were evoked.

where few visible responses were found despite extensive exploration (see also Mitz and Godschalk, 1989, their Fig. 2A).

The movements evoked from most stimulation sites did not differ in their characteristics from the movements reported by others using microstimulation in the MI (Kwan et al., 1978; McGuinness et al., 1980; Sessle and Wiesendanger, 1982; Waters et al., 1990; and others). These were brief joint rotations at a single joint, or a few adjacent joints, that began and ended timelocked to the stimulus. There were a few sites, all in the vicinity of the SMA, where evoked movements were slow to evolve, a characteristic that has been described in a previous microstimulation study of that area (Mitz and Wise, 1987).

Nearly $60 \%$ of all the stimulation sites in the two hemispheres (1993/3390) yielded movements at or below the maximum stimulus current $(60 \mu \mathrm{A})$. Of these 'active sites', $71.5 \%$ (1425/1993) were associated with simple movements: movements about a single joint or movements of a limited, circumscribed region of the face, torso, etc. Movements evoked from the remaining sites involved either 2 joints (226 sites), 3 joints ( 36 sites), or 4 joints ( 2 sites). Simultaneous movement of a forelimb joint and an orofacial movement was classified as a two joint movement. Case M58 was mapped sufficiently to assess the relative frequency of combined face/forelimb responses. Combined forelimb/face movements were evoked from the PM (38 sites), and once from the MI.
In both animals movement sites for the hindlimb, forelimb, and face were distributed in a medial-to-lateral progression along a region of cortex bounded by the fundi of the central and arcuate sulci (Fig. 3A). There is an essentially parallel medial-to-lateral progression of hindlimb, forelimb, and orofacial sites in MI and PM that starts from a hindlimb representation that appears to be common to the SMA as well as the MI and PM (Fig. 3A, right). The PM's orofacial and forelimb movement representations overlap more than its forelimb and hindlimb representations (Fig. 2, right, Fig. 3A, right). The MI has abrupt transitions among its body part representations. The abrupt transitions in the MI somatotopic areas is a universal feature of prior MI mapping studies (Woolsey et al., 1952; Sessle and Wiesendanger, 1982; Waters et al., 1990), but not of SMA studies (Mitz and Wise, 1987; Luppino et al., 1991).

Proximal and distal movement sites are largely intermixed in some parts of the PM forelimb representation, most notably on the precentral gyrus posterior and medial to the posterior tip of the arcuate (Fig. 3C). Laterally along the inferior limb, distal sites dominate. In one monkey (M58) only proximal limb movements were evoked from the medial wall of the arcuate spur; the other monkey (M57) had fewer proximal limb movement sites in general and did not have a clear proximal forelimb movement region. The assessment of proximal versus distal representations for the hindlimb is in- 
A
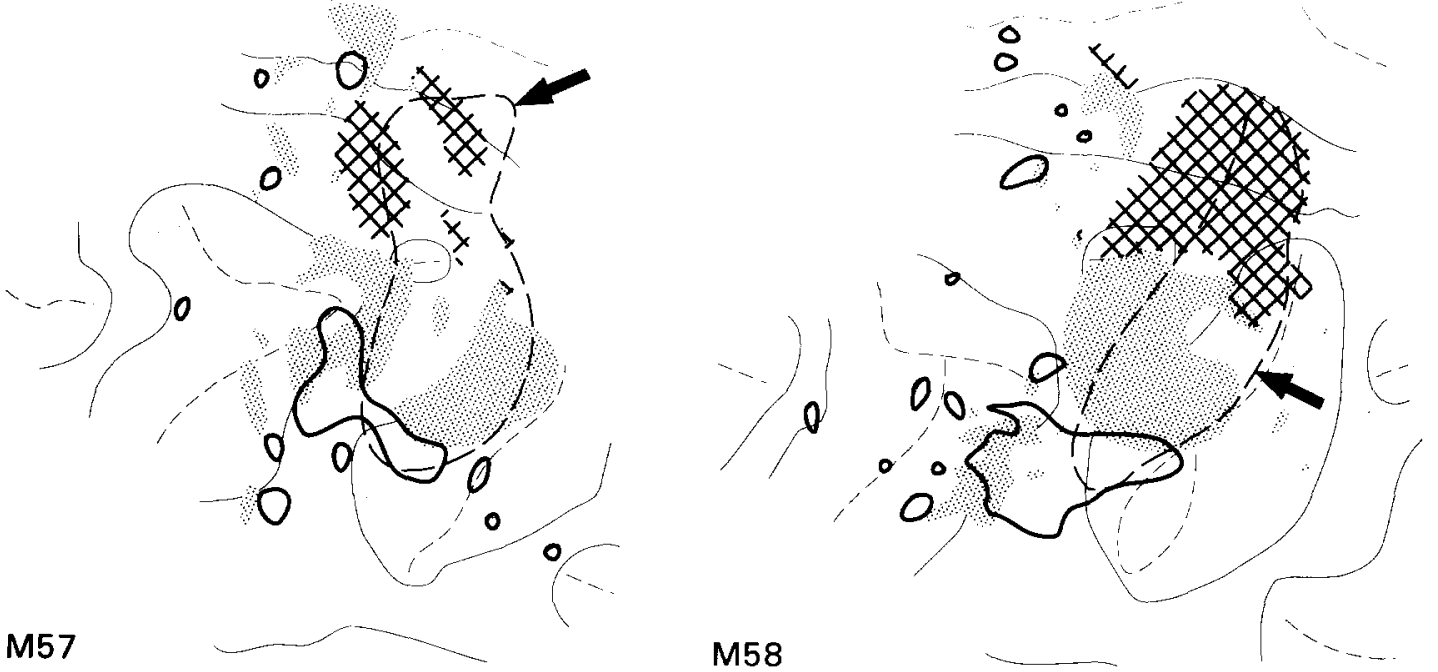

B
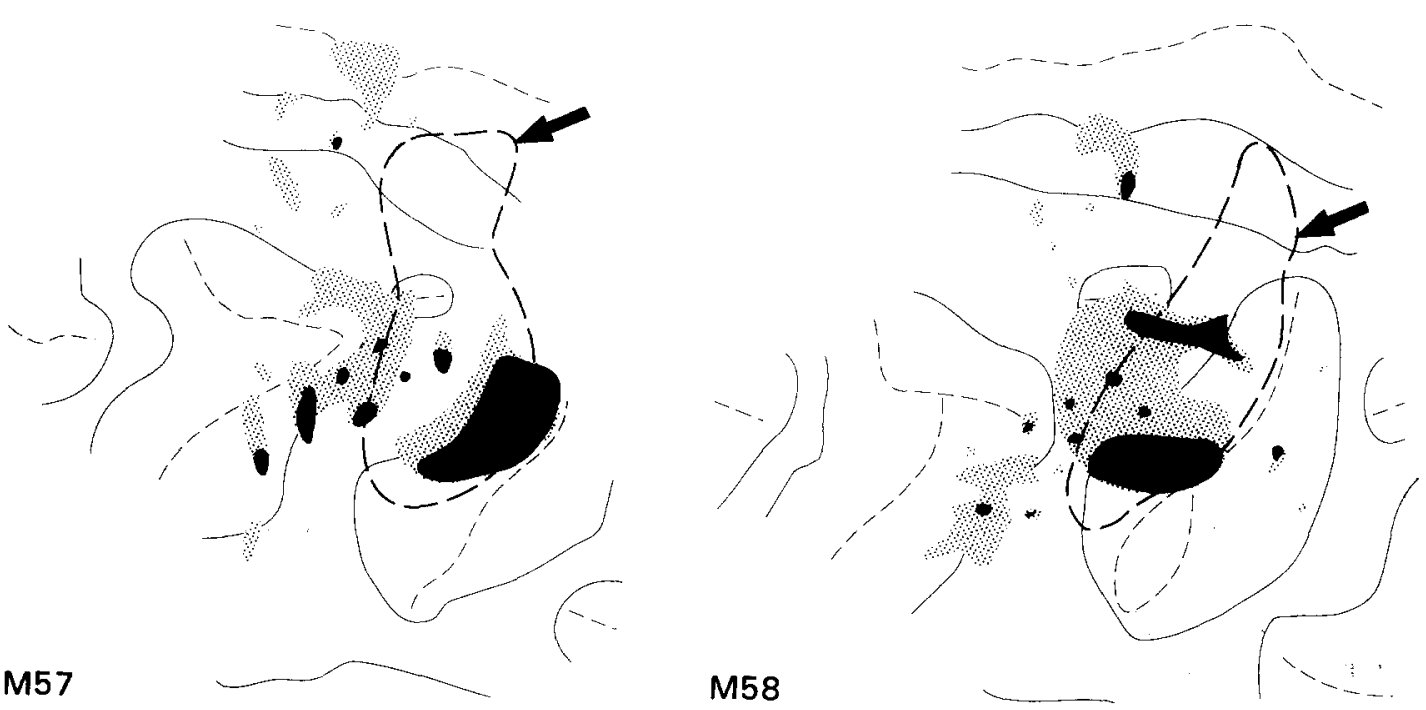

C
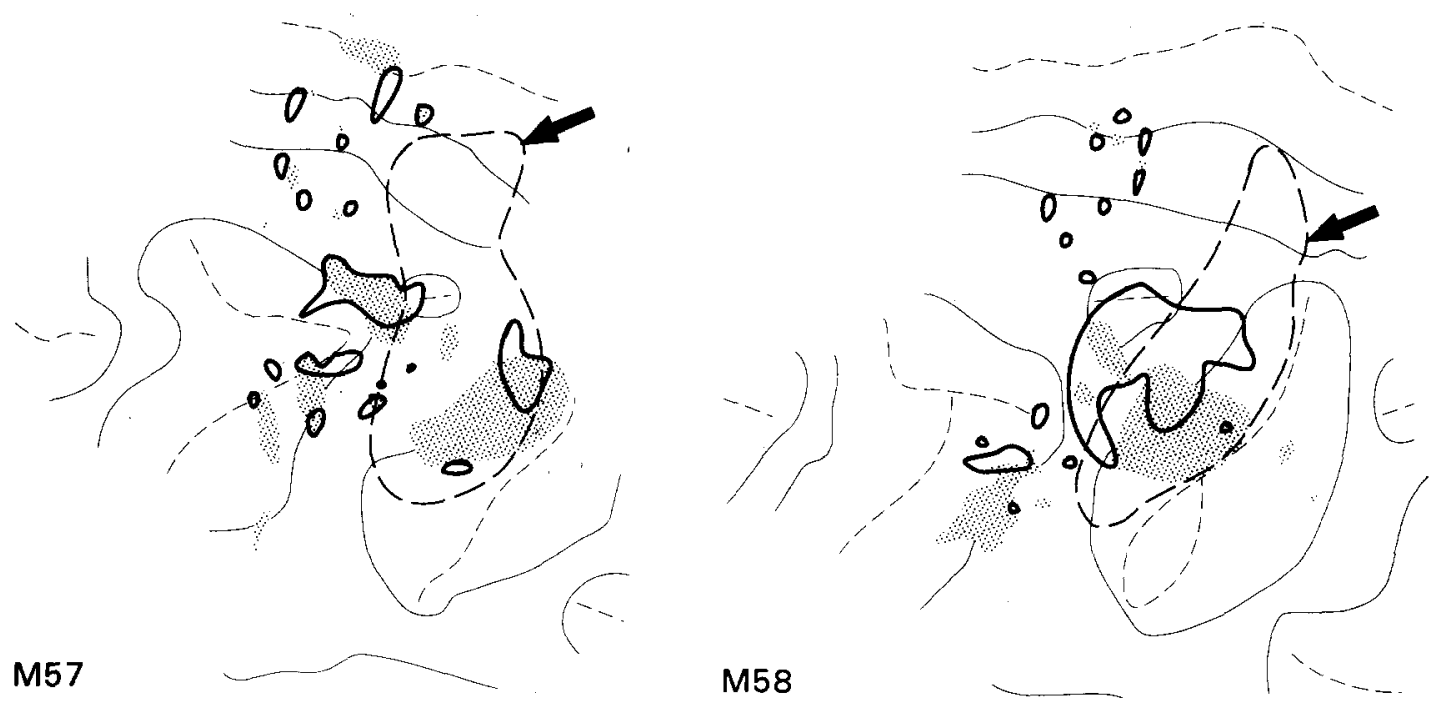
conclusive. No distal hindlimb movements were evoked from M57, presumably because the appropriate cortical region was not explored. In M58 foot movements were evoked from the caudomedial part of the hindlimb representation, but it is unclear whether these sites belong to the MI, PM, or SMA.

\subsection{Effects of stimulus current}

Low threshold responses common to the MI were rare in the PM. This is best examined by studying the forelimb representations where a clear MI/PM boundary exists. A significant proportion of the MI forelimb representation responsive to high stimulus currents $(11-50 \mu \mathrm{A}$ ) was also responsive to low currents ( $\leq 10$ $\mu \mathrm{A})$ (Fig. 3B). In the PM this proportion was far less, owing to the paucity of forelimb responses to low currents in general. The PMd was devoid of low threshold forelimb responses except for a few sites just at the border between the PM and MI (Fig 3B, right). The low threshold responses of the PMv were scattered, with no apparent organizational pattern.

\section{Discussion}

The PM has been subdivided anatomically in a number of different ways, based upon different criteria (Matelli et al., 1985; Barbas and Pandya, 1987; Dum and Strick, 1991; Luppino et al., 1991; Preuss and Goldman-Rakic, 1991). However, the paucity of agreement among these analyses, and disagreements over the bases of some of the proposed criteria (Huntley and Jones, 1991b), have led us to present our findings only in relation to the most commonly used anatomical criteria (see Methods). We found that the PM appears to have a single medial-to-lateral representation of the body oriented parallel to that of the MI (Fig. 3A), and similar to it except that there is greater overlap of forelimb and face representations in the PM than seen in the MI (Sessle and Wiesendanger, 1982; Waters et al., 1990; present study). Such a distinction in overlap extent did not emerge from comparisons of hindlimb and forelimb representations.

\subsection{Somatotopy}

Hashimoto et al. (1981) failed to identify separate forelimb and hindlimb representations in the PM using local field potentials in operantly-conditioned monkeys. Their result disagrees with the present results as well as with a number of other studies (Matsumura and Kubota, 1979; Muakkassa and Strick, 1979; Godschalk et al., 1984; Kurata et al., 1985; Kurata, 1989; Dum and Strick, 1991; Morecraft and Van Hoesen, 1992). Field potential recording may not have sufficient spatial resolution for discriminating separate limb representations, especially if the PM does have overlapping representations. Further, the present data show the hindlimb representation confined to a region medial to the precentral dimple; Hashimoto et al. apparently did not sample sites in that region, which might also explain their negative results.

The locations of the forelimb and hindlimb representations seen in the present data are in reasonably good agreement with single-unit recording (Kurata et al., 1985; Gentilucci et al., 1988; Rizzolatti et al., 1988; Kurata, 1989), and some aspects of the retrograde fiber tracing data (Matsumura and Kubota, 1979; Muakkassa and Strick, 1979; Godschalk et al., 1984; Morecraft and Van Hoesen, 1992). However, the present stimulation data differ from the observations by Muakkassa and Strick (1979), as do some other anatomical studies (Godschalk et al., 1984; Dum and Strick, 1991; Morecraft and Van Hoesen, 1992; He et al., 1993), in that no clear hindlimb representation could be found along the cortex of the superior limb of the arcuate sulcus. Despite the general agreement among the studies cited, the exact organization of the cortex in the area of the precentral sulcus remains unclear. He et al. looked for separate forelimb and hindlimb representations within the superior precentral sulcus, but their results were equivocal. We evoked a mixture of forelimb and hindlimb movements from this same region, and Kurata found both forelimb-specific and hindlimb-specific single-units (Kurata et al., 1985; Kurata, 1989).

Comparing the results of the present study with more detailed maps of the MI (Kwan et al., 1978; McGuinness et al., 1980; Sessle and Wiesendanger, 1982; Waters et al., 1990) and the SMA (Macpherson et al., 1982; Mitz and Wise, 1987), it appears that the monkey forelimb and hindlimb are each represented in a nearly continuous fashion across several motor areas of the frontal lobe. The stimulation-defined forelimb representation of the MI appears to merge anteriorly with the PM, and approaches, if not merges with, the forelimb representation of the SMA. The convexity of the cortex just medial to the superior limb of the arcuate sulcus, just lateral to the forelimb representation of the SMA, was relatively

Fig. 3. Somatotopic patterns. Orientation as in Fig. 1. Arrow in each drawing points to an outline of the region where Betz cells were identified (see Fig. 1). (A) The extent of orofacial, forelimb, and hindlimb representations in the cortex for currents at or below $50 \mu \mathrm{A}$. Orofacial areas are delineated by thick lines, forelimb areas by stippling, and hindlimb areas by cross-hatching. Note overlaps between representations. (B) Effects of stimulus current on the distribution of forelimb movement sites. The distribution of low threshold ( $\leq 10 \mu \mathrm{A}$ ) sites are shown with a solid fill; higher threshold sites $(11-50 \mu \mathrm{A})$ are shown with stippling. (C) Proximal versus distal forelimb movement sites for currents at or below $50 \mu \mathrm{A}$. Distal forelimb movement sites shown with stippling, proximal sites outlined with thick lines. 
inexcitable in both animals. This is also a region that has a paucity of corticospinal projections (He et al., 1993).

The present methods differ from previous, less successful efforts at PM microstimulation primarily in the selection of electrode tip geometry and stimulation parameters. The present study employed $100 \mathrm{~ms}$ pulse trains ( 30 pulses) rather than $30-36$ ms trains ( 11 pulses) more commonly used. In the MI the effect of the pulse train length on electromyographic activity becomes asymptotic at about $30 \mathrm{~ms}$ (Asanuma et al., 1976). Longer trains do not markedly alter MI thresholds for evoked movement using either surface stimulation (Lilly et al., 1952) or intracortical microstimulation (Kwan et al., 1978; Mitz, 1984). The somatotopic pattern of movements evoked from the hand area in the MI is essentially the same using either $30 \mathrm{~ms}$ pulse trains or $100 \mathrm{~ms}$ pulse trains (Mitz, 1984). In the SMA, however, the pulse train length has a significant impact on the likelihood of evoking a response (Mitz and Wise, 1987), presumably because the resulting temporal summation is necessary to activate less direct or less secure pathways to the motor neuron pools. It is possible that the pulse train duration was the primary reason that more PM movements were observed in the present study than in previous studies where shorter trains were employed (Weinrich and Wise, 1982; Wise and Mauritz, 1985; Gentilucci et al., 1988). On the other hand, the same prior studies also used standard recording electrodes with small exposed tips. Small electrode tips have a greater current density for a given stimulus current than larger tips, since current density is essentially the current divided by the effective surface area of the tip. At high stimulation currents the elevated current density can block the action potential conduction for nerve elements near the electrode tip. The conduction block arises from hyperpolarizing currents that must flow into the cell at sites away from the electrode tip as outward (depolarizing) currents flow towards the tip when the tip is negative. Conduction blockage has been demonstrated for both peripheral and central nerve fibers using currents of 2-10 times the fiber's threshold (Katz and Miledi, 1965; Jankowska and Roberts, 1972; Roberts and Smith, 1973; see Ranck, 1975 for review). Monopolar cathodal electrical stimulation at these currents produces a central core of inhibition and an outer shell of excitation. There are no comparable studies for stimulation of cortical gray matter, but it is possible that the larger tip exposures used in the present study were more effective because they avoided inhibitory effects near the electrode tip.

There is no reason to expect that passive current spread was a factor in producing larger cortical representations. The stimulation currents used in the present study were similar to those employed in previous studies (Weinrich and Wise (60 $\mu \mathrm{A}$ maximum), 1982; Wise and Mauritz (50 $\mu \mathrm{A}$ maximum), 1985; Gentilucci et al., 1988 (40 $\mu \mathrm{A}$ maximum)) and the maximum current spread in tissue does not depend upon the electrode tip geometry (Ranck, 1975).

The forelimb representation includes not only the MI, PM and SMA, but also passes without interruption from the SMA into parts of area 24 (Luppino et al., 1991; Morecraft and Van Hoesen, 1992). The stimulation-defined hindlimb representations for the MI, PM and SMA, and parts of area 24 appear to be clustered into a single medial representation without clear physiological boundaries, except for a greater segregation of proximal versus distal hindlimb sites in the MI (Woolsey et al., 1952; Macpherson et al., 1982; Mitz and Wise, 1987; Luppino et al., 1991; present study). The PM orofacial representation lies adjacent to and apparently contiguous with the MI face area. The frontal lobe orofacial motor representation is thus not continuous (or contiguous) like that for the two limbs, because the SMA orofacial representation does not join with the other two orofacial representations.

\subsection{Overlap}

The interpretation of 'overlapping' motor representations is limited by the methods employed. It is possible that the overlaps of adjacent representations, as presented in Figs. 2 and 3, are not from arbitrary interminglings of sites, but are from independent, uninterrupted representations that segregate at different cortical layers. Microstimulation data for both figures was collapsed across cortical layers and projected onto layer 5 (see Methods). In the chronic monkey preparation measurements of electrode depth and trajectory can lead to substantial reconstruction errors when made without verification by numerous marking lesions, or other anatomical methods. If the errors are too great they preclude using data from several different penetrations to reconstruct a series of sites radial to the layers of the cortex. In the present study electrode positional errors were about $0.5 \mathrm{~mm} / 10 \mathrm{~mm}$ of penetration depth (see Methods), too large for depth reconstructions. In addition, there were relatively few opportunities to attempt track reconstructions. Without radial stimulation profiles we cannot distinguish between random intermixing and segregation of sites by cortical depth. In the orofacial and forelimb regions of the MI, and adjacent premotor cortex, even the finest details of somatotopic organization change very little as a function of cortical depth (Asanuma and Rosén, 1972; Murphy et al., 1978; McGuinness et al., 1980; Murray and Sessle, 1992). Comparatively little is known about the remaining nonprimary motor areas. The pyramidal tract projection, which originates from a single cortical layer (Jones and Wise, 1977), dominates the microstimulation-evoked responses of the MI (Mitz and Humphrey, 1986). The pyramidal tract may not be the dominant pathway for evoked responses from the PM or SMA; a cortico- 
cortico-spinal pathway via the MI (Pandya and Kuypers, 1969; Matsumura and Kubota, 1979; Muakkassa and Strick, 1979; (Godschalk et al., 1984), for example, could be the major contributor. In the only anatomical study where forelimb, hindlimb and face representations were evaluated within the same subject, there was no evidence for segregation of representations by cortical layer in either the PM or SMA (Godschalk et al., 1984).

Whether overlap is by intermixing or by cortical layering, within the MI there is relatively little overlap between adjacent representations (Woolsey et al., 1952), and the transition from the orofacial representation to the forelimb representation is especially abrupt (Aizawa et al., 1990; Waters et al., 1990; Huntley and Jones, 1991a; Donoghue et al., 1992; present study). The segregation between the hindlimb and forelimb representations in the PM is similar to that of the MI. The region from which movements of both limbs could be evoked was limited to the superior precentral sulcus (Fig. 3A) in both cases studied. However, the amount of overlap between PM orofacial and forelimb representations is comparatively large. In subject M58, intermixing of orofacial and forelimb movement sites occurs in the caudal bank of the inferior limb of the arcuate sulcus, and in the adjacent hemispheric convexity (Fig. 3A). The map in this animal was complete enough to quantify the commonality of forelimb/face sites in the PM ( 38 sites) compared to the MI (1 site). In subject M57, where fewer orofacial movements were evoked from the cortex in the arcuate sulcus, the orofacial/forelimb overlap is still clearly greater in the PM than the MI (Fig. 3A). Since it is more difficult to evoke movements from the PM compared to the MI (see Introduction), a priori one expects overlap between movement representations to be more grossly underrepresented in the PM than the MI. Overlap of orofacial and forelimb movement sites has also been observed using standard microstimulation in lateral area 6 (M.-C. Hepp-Reymond, personal communication), where some of the neurons are active during force-modulation in a precision grip task (HeppReymond et al., 1994).

The results of the present study place the subdivisions of the PM into the broader perspective of PM somatotopy. PMd somatotopy may be more discretely organized than single-unit recordings would suggest (Kurata, 1989); the forelimb representation does not substantially overlap with the hindlimb representation, which is located more medially and caudally. PMv somatotopy is quite different. Broad portions of the PMv have either mutual or at least intermixed access to the motor apparatus of toth forelimb and face, a finding that is in good agreement with the series of studies by Rizzolatti and his colleagues (Rizzolatti et al., 1981; Gentilucci et al., 1983, 1988), and more recently by Graziano and Gross (1992, 1994). Other, largely unex- plored, regions of the PMv have segregated hand and face outputs; perhaps these regions of the PM have their own, specialized functional roles.

\section{Acknowledgments}

The authors wish to thank Els de Jong, Dr. David S. Pomerantz, Dr. David Mok, and Dr. Th. Stijnen for their contributions to data collection and analysis, Dr. Driss Boussaoud for the cortex unfolding methodology, and Eddy Dalm and Erika Sabel-Goedknecht for surgery and tissue preparation. This research was supported in part by NATO Collaborative Research Grant No. 299.88 .

\section{References}

Aizawa, H., Mushiake, H., Inase, M. and Tanji, J. (1990) An output zone of the monkey primary motor cortex specialized for bilateral hand movement. Exp. Brain Res., 82: 219-221.

Asanuma, H. and Rosén, I. (1972) Topographical organization of cortical efferent zones projecting to distal forelimb muscles in the monkey. Exp. Brain Res., 14: 243-256.

Asanuma, H., Arnold, A., Zarzecki, P. (1976) Further study on the excitation of pyramidal tract cells by intracortical microstimulation. Exp. Brain Res., 26: 442-461.

Barbas, H. and Pandya, D.N. (1987) Architecture and frontal cortical connections of the premotor cortex (area 6) in the rhesus monkey. J. Comp. Neurol., 256: 211-288.

Di Pellegrino, G. and Wise, S.P. (1991) A neurophysiological comparison of three distinct regions of the primate frontal lobe. Brain, 114: 951-978.

Donoghue, J.P., Leibovic, S. and Sanes, J.N. (1992) Organization of the forelimb area in squirrel monkey motor cortex: representation of digit, wrist, and elbow muscles. Exp. Brain Res., 89: 1-19.

Dum, R.P. and Strick, P.L. (1991) The origin of corticospinal projections from the premotor areas in the frontal lobe. J. Neurosci., 3: 667-689.

Fogassi, L., Gallese, V., di Pellegrino, G., Fadiga, L., Gentilucci, M., Luppino, G., Matelli, M., Pedotti, A. and Rizzolatti, G. (1992) Space coding by premotor cortex. Exp. Brain Res. 89: 686-690.

Gentilucci, M., Scandolara, C., Pigarev, I.N. and Rizzolatti, G. (1983) Visual responses in the postarcuate cortex (area 6) of the monkey that are independent of eye position. Exp. Brain Res., 50: 464-468.

Gentilucci, M., Fogassi, L., Luppino, G., Matelli, M., Camarda, R. and Rizzolatti, G. (1988) Functional organization of inferior area 6 in the macaque monkey. I. Somatotopy and the control of proximal movements. Exp. Brain Res., 71: 475-490.

Godschalk, M., Lemon, R.N., Kuypers, H.G.J.M. and Ronday, H.K. (1984) Cortical afferents and efferents of monkey postarcuate area: an anatomical and electrophysiological study. Exp. Brain Res., 56: 410-424.

Godschalk, M., Mitz, A.R., Van der Burg, J. and Van Duin, B. (1990) Microstimulation map of the monkey premotor cortex. Soc. Neurosci. Abstr., 16: 1133.

Gottlieb, J.P., Bruce, C.J. and MacAvoy, M.G. (1993) Smooth eye movements elicited by microstimulation in the primate frontal eye field. J. Neurophysiol., 69: 786-799.

Graziano, M.S.A. and Gross, C.G. (1992) Coding of extrapersonal visual space in body-part centered coordinates. Soc. Neurosci. Abstr., 18: 593.

Graziano, M.S.A. and Gross, C.G. (1994) Mapping visual space around the arm with bimodal visual-tactile neurons. Soc. Neurosci. Abstr., 20: 1278. 
Hashimoto, S., Gemba, H. and Sasaki, K. (1981) Distribution of slow cortical potentials preceding self-paced hand and hindlimb movements in the premotor and motor areas of monkeys. Brain Res., 224: 247-259.

He, S.-Q., Dum, R.P. and Strick, P.L. (1993) Topographic organization of corticospinal projections from the frontal lobe: motor areas on the lateral surface of the hemisphere. J. Neurosci., 13: 952-980

Hepp-Reymond, M.-C., Husler, E.J., Maier, M.A. and Qi, H.-X. (1994) Force-related neuronal activity in two regions of the primate ventral premotor cortex. Can. J. Physiol. Pharmacol., 72: 571-579.

Huerta, M.F. and Kaas, J.H. (1990) Supplementary eye field as defined by intracortical microstimulation: connections in macaques. $\mathbf{J}$. Comp. Neurol. 293: 299-330.

Humphrey, D.R. and Reed, D.J. (1983) Separate cortical system for control of joint movement and joint stiffness: reciprocal activation and coactivation of antagonist muscles. In: J.E. Desmedt (Ed.), Motor Control Mechanisms in Health and Disease, Raven Press, New York, pp. 347-372.

Huntley, G.W. and Jones, E.G. (1991a) Relationship of intrinsic connections to forelimb movement representations in monkey motor cortex: a correlative anatomic and physiological study. J. Neurphysiol., 66: 390-413.

Huntley, G.W. and Jones, E.G. (1991b) The emergence of architectonic field structure and areal borders in developing monkey sensorimotor cortex. Neuroscience, 44: 287-310.

Jankowska, E. and Roberts, W.J. (1972) An electrophysiological demonstration of the axonal projections of single spinal interneurones in the cat. J. Physiol. (Lond.), 222: 597-622.

Jones, E.G. and Wise, S.P. (1977) Size, laminar and columnar distribution of efferent cells in the sensory-motor cortex of monkeys. J. Comp. Neurol., 175: 391-437.

Katz, B. and Miledi, R. (1965) Propagation of electrical activity in motor nerve terminals. Proc. R. Soc. Lond. B., 161: 453-482.

Kurata, K. (1989) Distribution of neurons with set- and movementrelated activity before hand and foot movements in the premotor cortex of rhesus monkeys. Exp. Brain Res., 77: 245-256.

Kurata, K. (1993) Premotor cortex of monkeys: set- and movementrelated activity reflecting amplitude and direction of wrist movements. J. Neurophysiol. 69: 187-200.

Kurata, K., Okano, K. and Tanji, J. (1985) Distribution of neurons related to a hindlimb as opposed to forelimb movement in the monkey premotor cortex. Exp. Brain Res., 60: 188-191.

Kwan, H.C., MacKay, W.A., Murphy, J.T. and Wong, Y.C. (1978) Spatial organization of precentral cortex in awake primates. II. Motor outputs. J. Neurophysiol., 41: 1120-1131.

Lemon, R. (1984) Methods for Neuronal Recording in Conscious Animals, IBRO Handbook Series, Vol. 4, Wiley, New York, pp. 56-60.

Lilly, J.C., Austin, G.M. and Chambers, W.W. (1952) Threshold movements produced by excitation of cerebral cortex and efferent fibers with some parametric regions of rectangular current pulses (cats and monkeys). J. Neurophysiol., 15: 319-341.

Luppino, G., Matelli, M. and Rizzolatti, G. (1990) Cortico-corticai connections of two electrophysiologically identified arm representations in the mesial agranular frontal cortex. Exp. Brain Res., 82: 214-218.

Luppino, G., Matelli, M., Camarda, R.M., Gallese, V. and Rizzolatti, G. (1991) Multiple representations of body movements in mesial area 6 and the adjacent cingulate cortex: an intracortical microstimulation study in the macaque monkey. J. Comp. Neurol., 311: 463-482.

Macpherson, J.M., Marangoz, C., Miles, T.S. and Wiesendanger, M. (1982) Microstimulation of the supplementary motor area (SMA) in the awake monkey. Exp. Brain Res., 45: 410-416.

Matelli, M., Luppino, G. and Rizzolatti, G. (1985) Patterns of cytochrome oxidase activity in the frontal agranular cortex of the macaque monkey. Behav. Brain Res., 18: 125-136.

Matelli, M., Luppino, G. and Rizzolatti, G. (1991) Architecture of su- perior and mesial area 6 and the adjacent cingulate cortex in the macaque monkey. J. Comp. Neurol., 311: 445-462.

Matsumura, M. and Kubota, K. (1979) Cortical projection to handarm motor area from post-arcuate area in macaque monkeys: a histological study of retrograde transport of horseradish peroxidase. Neurosci. Lett., 11: 241-246.

McGuinness, E., Sivertsen, D. and Allman, J.M. (1980) Organization of the face representation in macaque motor cortex. J. Comp. Neurol., 193: 591-608.

Mitz, A.R. (1984) Microstimulation mapping of the primate motor cortex: changes in topography after pyramidotomy and after subsequent motor recovery. Doctoral dissertation, Emory University Department of Physiology, Atlanta, Georgia, 233 pp.

Mitz, A.R. and Godschalk, M. (1989) Eye-movement representation in the frontal lobe of rhesus monkeys. Neurosci. Lett., 106: 157-162.

Mitz, A.R. and Humphrey, D.R. (1986) Intracortical stimulation in pyramidotomized monkeys. Neurosci. Lett., 64: 59-64.

Mitz, A.R. and Wise, S.P. (1987) The somatotopic organization of the supplementary motor area: intracortical microstimulation mapping. J. Neurosci., 7: 1010-1021.

Mitz, A.R., Reed, D.J. and Humphrey, D.R. (1984) A sequential pulse generator for producing true biphasic stimuli. Electroenceph. Clin. Neurophysiol., 57: 587-590.

Morecraft, R.J. and Van Hoesen, G.W. (1992) Cingulate input to the primary and supplementary motor cortices in the rhesus monkey: evidence for somatotopy in areas $24 \mathrm{c}$ and $23 \mathrm{c}$. J. Comp. Neurol., 322: 471-489.

Muakkassa, K.F. and Strick, P.L. (1979) Frontal lobe inputs to primate motor cortex: evidence for four somatotopically organized 'premotor' areas. Brain Res., 177: 176-182.

Mushiake, H., Inase, M. and Tanji, J (1991) Neuronal activity in the primate premotor, supplementary, and precentral motor cortex during visually guided and internally determined sequential movements. J. Neurophysiol., 66: 705-718.

Murphy, J.T., Kwan, H.C., MacKay, W.A. and Wong, Y.C. (1978) Spatial organization of precentral cortex in awake primates. III. Input-output coupling. J. Neurophysiol., 41: 1132-1139.

Murray, G.M. and Sessle, B.J. (1992) Functional properties of single neurons in the face primary motor cortex of the primate. I. Input and output features of tongue motor cortex. J. Neurophysiol., 67: 747-758.

Pandya, D.N. and Kuypers, H.G.J.M. (1969) Cortico-cortical connections in the rhesus monkey. Brain Res., 13: 13-36.

Preuss, T.M. and Goldman-Rakic, P.S. (1991) Myelo- and cytoarchitecture of the granular frontal cortex and surrounding regions in the strepsirhine primate Galago and the anthropoid primate Macaca. J. Comp. Neurol., 310: 429-474.

Ranck, J.B. (1975) Which elements are excited in electrical stimulation of mammalian central nervous system: A review. Brain Res., 98: 417-440.

Rizzolatti, G., Scandolara, C., Matelli, M. and Gentilucci, M. (1981) Afferent properties of periarcuate neurons in macaque monkeys. II. Visual responses. Behav. Brain Res., 2: 147-163.

Rizzolatti, G., Camarda, R., Fogassi, L., Gentilucci, M., Luppino, G. and Matelli, M. (1988) Functional organization of inferior area 6 in the macaque monkey. II. Area F5 and the control of distal movements. Exp. Brain Res., 71: 491-507.

Roberts, W.J. and Smith, D.O. (1973) Analysis of threshold currents during microstimulation of fibres in the spinal cord. Acta. Physiol. Scand., 89: 384-394.

Schlag, J. and Schlag-Rey, M. (1987) Evidence for a supplementary eye field. J. Neurophysiol. 57: 179-200.

Sessle, B.J. and Wiesendanger, M. (1982) Structural and functional definition of the motor cortex in the monkey (Macaca fascicularis). J. Physiol., 323: 245-266.

Shima, K. and Tanji, J. (1994) Neuronal activity in the supplementary 
and pre-supplementary motor area in relation to sequential performance of multiple movemerits. Soc. Neurosci. Abstr., 20: 986.

Shima, K., Aya, K., Mushiakt, H. Inase, M., Aizawa, H. and Tanji, J. (1991) Two movement-related foci in the primate cingulate cortex observed in signal-triggered and self-paced forelimb movements. J. Neurophysiol., 65: 188-202.

Strick, P.L. and Preston, J.B. (1982a) Two representations of the hand in area 4 of a primate. I. Motor output organization. J. Neurophysiol. 48: 139-149.

Strick, P.L. and Preston, J.B. (1982b) Two representations of the hand in area 4 of a primate. II. Somatosensory input organization. J. Neurophysiol. 48: 150-159.

Van Essen, D.C. and Maunsell, J.H.R. (1980) Two-dimensional maps of the cerebral cortex. J. Comp. Neurol., 191: 255-281.

Waters, R.S., Samulack, D.D., Dykes, R.W. and McKinley, P.A. (1990) Topographic organization of baboon primary motor cortex: face, hand, forelimb, and shoulder representation. Somatosens. Motor Res., 7: 485-514.
Weinrich, M. and Wise, S.P. (1982) The premotor cortex of the monkey. J. Neurosci., 2: 1329-1345.

Wise, S.P. and Mauritz, K.-H. (1985) Set-related neuronal activity in the premotor cortex of rhesus monkeys: effects of changes in motor set. Proc. R. Soc. Lond., 223: 331-354.

Wise, S.P. and Tanji, J. (1981) Supplementary and precentral motor cortex: contrast in responsiveness to peripheral input in the hindlimb area of the unanesthetized monkey. J. Comp. Neurol., 195: 433-451.

Woolsey, C.N., Settlage, P.H., Meyer, D.R., Sencer, W., Pinto Hamuy, T. and Travis, A.M. (1952) Patterns of localization in precentral and 'supplementary' motor areas and their relation to the concept of a premotor area. Res. Publ. Assoc. Nerv. Ment. Dis., 30: 238-264.

Woolsey, C.N., Erickson, T.C. and Gilson, W.E. (1979) Localization in somatic sensory and motor areas of human cerebral cortex as determined by direct recording of evoked potentials and electrical stimulation. J. Neurosurg., 51: 476-506. 